\title{
Concurrent renal cell carcinoma and urothelial carcinoma: long-term follow-up study of 27 cases
}

Nienie $\mathrm{Qi}^{1+}$, Yue Chen ${ }^{1 \dagger}$, Kan Gong ${ }^{2,3,4}$ and Hailong $\mathrm{Li}^{i^{*}}$

\begin{abstract}
Background: To investigate the clinical manifestation, diagnosis, treatment, and outcome of simultaneous occurrence of renal cell carcinoma (RCC) and urothelial carcinoma.

Methods: Twenty-seven consecutive patients with synchronous renal cell carcinoma and urothelial carcinoma treated in two tertiary medical centers from March 2005 to December 2015 were retrospectively reviewed. Their clinical, pathological, and prognostic features were evaluated. Kaplan-Meier curves were used to estimate overall survival.

Results: The median age was 69 years (range, 37-79 years). Seventeen patients presented with macroscopic hematuria, and 10 patients were asymptomatic. B-ultrasound, computed tomography (CT), and cystoscopy initially indicated RCC concurrent with ipsilateral upper tract urothelial carcinoma (UTUC) in 5 cases, RCC concurrent with contralateral UTUC in 1 case, RCC concurrent with bladder tumor in 17 cases, RCC concurrent with both ipsilateral UTUC and bladder tumor in 1 case, RCC in 2 cases and ureter carcinoma in 1 case. Different treatments were performed. The median follow-up time after surgery was 23 months. For patients with synchronous RCC and bladder tumor, there was no significant survival difference between patients treated with partial nephrectomy and radical nephrectomy. During follow up, four patients died of RCC, three patients died of non-oncological disease, one patient died of ureter carcinoma. The 3-year overall survival rate was $80.8 \%$.
\end{abstract}

Conclusions: Concurrence of RCC and urothelial carcinoma is clinically rare. Treatments should be individualized. The prognosis for a patient with synchronous RCC and urothelial carcinoma is possibly associated with the more aggressive one.

Keywords: Renal cell carcinoma, Urothelial carcinoma, Treatment, Prognosis

\section{Background}

Renal cell carcinoma (RCC) and urothelial carcinoma are both common urological malignancies. Their simultaneous occurrence in a patient is, however, extraordinarily rare. In the English-language literature, the first case was reported by Graves and Templeton in 1921 [1]. Most studies of synchronous RCC and urothelial carcinoma have been described in case report or small series. Due to the rarity of the disease and the limited reports

* Correspondence: 1102485781@qq.com

${ }^{\dagger}$ Equal contributors

${ }^{1}$ Department of Urology, The Affiliated Hospital of Xuzhou Medical

University, Xuzhou, China

Full list of author information is available at the end of the article in the literature, the treatment and outcomes of concurrent RCC and urothelial carcinoma are still uncertain, especially for patients with concurrent RCC and bladder tumor.

In this study, we carried out a long-term follow-up of 27 cases in 2 tertiary medical centers (Peking University First Hospital and The Affiliated Hospital of Xuzhou Medical University). To the best of our knowledge, this is the largest series of concurrent renal cell carcinoma and urothelial carcinoma ever reported in English literature.

\section{Methods}

Twenty-seven consecutive patients with synchronous RCC and urothelial carcinoma were retrospectively reviewed in this study. Informed consent was obtained 
from all participants. All the patients were diagnosed and treated between March 2005 and December 2015. Demographic data, including age, gender, smoking history, symptom, tumor location and size, treatment, postoperative pathological finding, adjuvant therapy, recurrence, and survival status were collected. Tumor stage was assessed according to the 2009 AJCC staging system. Tumor grade was assessed according to the WHO classification of 2004. For patients with synchronous RCC and bladder tumor, the effect of surgical type on survival was assessed by the Kaplan-Meier method and differences among survival curves were tested by the log-rank test. Among these patients, only two cases were treated with radical nephroureterectomy (RNU), so we combined these two cases with cases treated by radical nephrectomy ( $\mathrm{RN})$ as one group for analysis. All procedures performed in studies involving human participants were in accordance with the ethical standards of the institutional and national research committee and with the 1964 Helsinki Declaration and its later amendments or comparable ethical standards. The study was approved by the institutional review board from Peking University First Hospital and The Affiliated Hospital of Xuzhou Medical University.

\section{Results}

\section{Clinical features}

A total of 27 patients were involved in this study and patients' characteristics are demonstrated in Table 1. The median age was 69 years (range, 37-79 years). Most of them were men. Thirteen patients $(48.1 \%)$ had a smoking history. The main symptom was macroscopic hematuria, accompanied by irritative symptoms or backache. Ten patients were asymptomatic and detected by regular physical examinations. Clinical diagnosis of concurrent RCC and urothelial carcinoma was made in 24 patients (Figs. 1 and 2). Two patients were initially diagnosed as isolated RCC, and one patient was initially diagnosed as isolated ureter carcinoma.

\section{Treatment and pathological findings}

Table 2 reveals operative parameters, pathological findings, and follow-up results. Twenty-six patients underwent onestage surgery, one patient underwent partial nephrectomy (PN) at first surgery due to the poor function of the contralateral kidney, and radical cystectomy (RC) was operated 1 month later. Different treatments were performed according to the tumor. Five patients diagnosed with RCC and ipsilateral upper tract urothelial carcinoma (UTUC) underwent radical nephroureterectomy; one patient diagnosed with RCC and ipsilateral UTUC and bladder tumor underwent RNU and transurethral resection of bladder tumor (TURBT); one patient diagnosed with RCC and contralateral ureter carcinoma underwent $\mathrm{PN}$ and
Table 1 Patient and tumor demographics

\begin{tabular}{|c|c|}
\hline & $n=27$ \\
\hline Age (years), median (range) & $69(37-79)$ \\
\hline \multicolumn{2}{|l|}{ Sex, $n(\%)$} \\
\hline Male & $22(81.5 \%)$ \\
\hline Female & $5(18.5 \%)$ \\
\hline \multicolumn{2}{|l|}{ Smoke } \\
\hline Yes & $13(48.1 \%)$ \\
\hline No & $14(51.9 \%)$ \\
\hline \multicolumn{2}{|l|}{ Symptom } \\
\hline Hematuria & $17(63.0 \%)$ \\
\hline No & $10(37.0 \%)$ \\
\hline \multicolumn{2}{|l|}{ UC location } \\
\hline UTUC & $9(33.3 \%)$ \\
\hline BT & $16(59.3 \%)$ \\
\hline UTUC + BT & $2(7.4 \%)$ \\
\hline RCC size $(\mathrm{cm})$, median (range) & $3.5(0.6-14.5)$ \\
\hline \multicolumn{2}{|l|}{ RCC stage } \\
\hline $\mathrm{T} 1$ & $21(77.8 \%)$ \\
\hline $\mathrm{T} 2$ & $4(14.8 \%)$ \\
\hline T3 & $2(7.4 \%)$ \\
\hline \multicolumn{2}{|l|}{ RCC grade } \\
\hline G1 & $8(29.6 \%)$ \\
\hline $\mathrm{G} 2$ & $16(59.3 \%)$ \\
\hline G3 & $3(11.1 \%)$ \\
\hline \multicolumn{2}{|l|}{ UC stage } \\
\hline $\mathrm{Ta} / \mathrm{T} 1$ & $18(66.7 \%)$ \\
\hline T2 & $4(14.8 \%)$ \\
\hline T3 & $3(11.1 \%)$ \\
\hline $\mathrm{T} 2(\mathrm{UTUC})+\mathrm{T} 1(\mathrm{BT})$ & $1(3.7 \%)$ \\
\hline T1(UTUC) + T1(BT) & $1(3.7 \%)$ \\
\hline \multicolumn{2}{|l|}{ Number of tumor } \\
\hline 2 & $21(77.8 \%)$ \\
\hline 3 & $4(14.8 \%)$ \\
\hline 4 & $2(7.4 \%)$ \\
\hline \multicolumn{2}{|l|}{ Hydronephrosis } \\
\hline Yes & $7(25.9 \%)$ \\
\hline No & $20(74.1 \%)$ \\
\hline Distant metastases, $n$ (\%) & $2(7.4 \%)$ \\
\hline
\end{tabular}

UC urothelial carcinoma, $R C C$ renal cell carcinoma, UTUC upper tract urothelial carcinoma, $B T$ bladder tumor

contralateral ureteroureterostomy. Two patients diagnosed with metastatic renal cell carcinoma were treated with cytoreductive RN. Postoperative pathology showed these two cases were synchronous renal cell carcinoma and urothelial carcinoma in the renal pelvis. The two kinds of tumors were not intermingled, but rather 


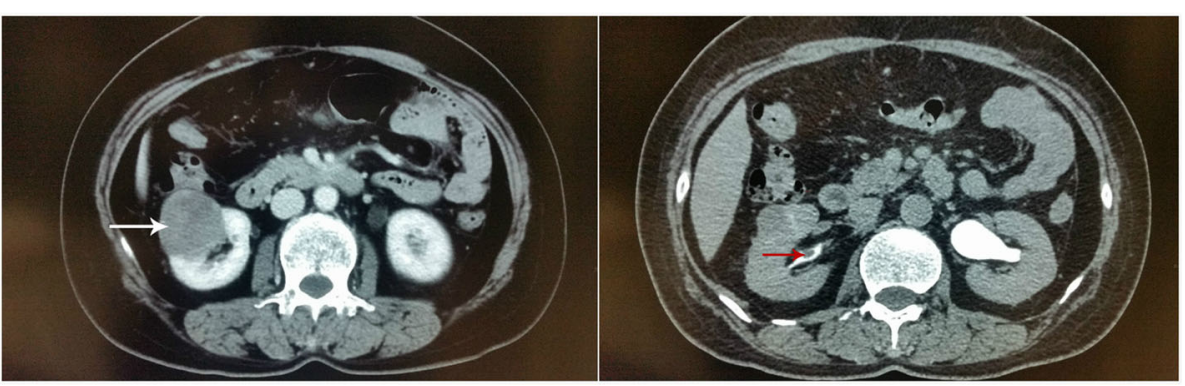

Fig. $1 \mathrm{CT}$ of a patient with concurrent renal cell carcinoma and ipsilateral pelvic carcinoma. The white arrow shows a renal mass in the right kidney; the red arrow shows a filling defect in the right renal pelvis

separated by normal renal parenchyma, neither of the patient received further ureterectomy; one patient diagnosed with ureter carcinoma was treated with RNU, and pathological examination revealed the coexistence of ureter carcinoma and renal cell carcinoma. For patients diagnosed with RCC and bladder tumor two patients were treated with RNU and TURBT and ureter carcinoma was postoperatively found in one patient. Ten patients were treated with PN and TURBT of RC. Five patients were treated with RN and TURBT or RC. Most of the histological types of RCC were clear cell renal cell carcinomas.

\section{Tumor recurrence and follow-up}

All the patients were regularly followed up with a median time of 23 months. During follow up, fossa recurrence of RCC appeared in one patient, bone metastasis of RCC appeared in two patients, liver metastasis of RCC appeared in one patient, and lung metastasis of RCC appeared in one patient. Bladder tumor recurrence appeared in six patients, and bone metastasis of UTUC appeared in one patient. All of the recurrence and metastasis were proved by the pathological results of surgery or biopsy. For the patient who underwent PN and contralateral ureteroureterostomy, bladder tumor occurred 1 year after initial surgery and TURBT was subsequently performed.

Seventeen patients $(63.0 \%)$ survived without tumors, two patients $(7.4 \%)$ survived with tumors, and eight patients (29.6\%) died during the follow-up. Four patients died of RCC progress or metastases, one patient emerged with ureter carcinoma metastasis and died 3 month later, and three patients died of non-oncological disease. For patients with synchronous RCC and bladder tumor, there was no significant survival difference between patients treated with partial nephrectomy or radical nephrectomy $(P=0.874)$. The 3 -year overall survival rate of all patients was $80.8 \%$.

\section{Discussion}

RCC represents $3 \%$ of adult cancers. Transitional cell carcinoma of the renal pelvic or ureter accounts for $5-7 \%$ of all urinary tract tumors and bladder cancer is the 11th most commonly diagnosed cancer in the world [2]. However, diagnosis of synchronous primary genitourinary tumors is uncommon. The increasing prevalence of genitourinary tumors has led to a reasonably increased diagnosis of synchronous tumors. About 50 cases of synchronous renal cell carcinoma and renal pelvic carcinoma have been reported in the literature [3-10].

The symptoms of synchronous RCC and urothelial carcinoma are similar to the solitary RCC or urothelial carcinoma of the urinary tract. In our study, hematuria was presented in 17 cases (63.0\%). Of patients, $48.1 \%$ were smokers, higher than reported in literature [7]. Concurrent renal cell carcinoma and ureter carcinoma were reported in a chronic hemodialysis patient, which

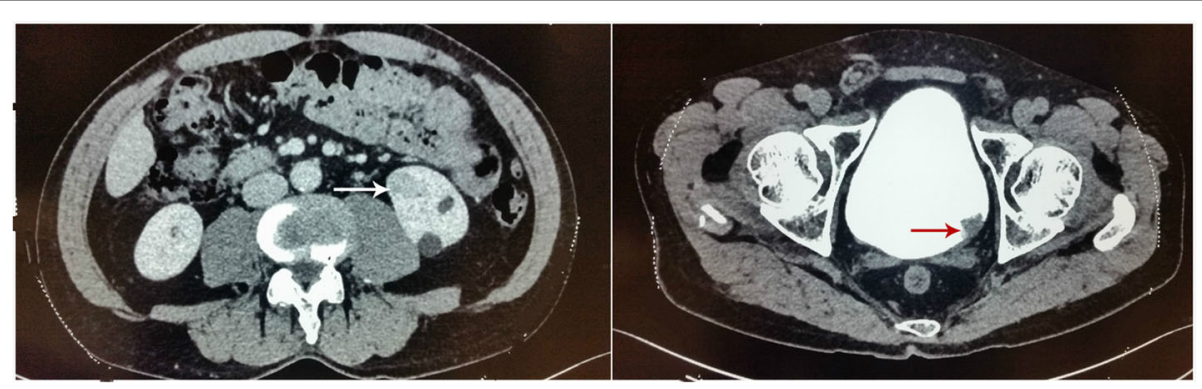

Fig. $2 \subset T$ of a patient with concurrent renal cell carcinoma and bladder tumor. The white arrow shows a renal mass in the left kidney; the red arrow shows a filling defect in the left posterior wall of bladder 
Table 2 Operative parameters and follow-up results

\begin{tabular}{|c|c|}
\hline & $n=27$ \\
\hline \multicolumn{2}{|l|}{ Operation type } \\
\hline RNU & 6 \\
\hline $\mathrm{RN}$ & 2 \\
\hline RNU + TURBT & 3 \\
\hline PN + TURBT & 9 \\
\hline RN + TURBT & 4 \\
\hline $\mathrm{PN}+$ ureteroureterostomy & 1 \\
\hline$P N+R C$ & 1 \\
\hline $\mathrm{RN}+\mathrm{RC}$ & 1 \\
\hline \multicolumn{2}{|l|}{ RCC histologic subtype, $n$ (\%) } \\
\hline cCRCC & $23(85.2 \%)$ \\
\hline $\mathrm{pRCC}$ & $2(7.4 \%)$ \\
\hline chRCC & $1(3.7 \%)$ \\
\hline Unclassified RCC & $1(3.7 \%)$ \\
\hline \multicolumn{2}{|l|}{ Adjuvant therapy } \\
\hline Instillation therapy & 8 \\
\hline Radiotherapy & 1 \\
\hline Follow-up (month), median (range) & $23(0.5-70)$ \\
\hline \multicolumn{2}{|l|}{ Recurrence } \\
\hline $\mathrm{RCC}$ & 5 \\
\hline Urothelial carcinoma & 7 \\
\hline \multicolumn{2}{|l|}{ Outcome, n (\%) } \\
\hline Alive & $19(70.4 \%)$ \\
\hline Dead & $8(29.6 \%)$ \\
\hline
\end{tabular}

$R N U$ radical nephroureterectomy, TURBT transurethral resection of bladder tumor, $P N$ partial nephrectomy, $R N$ radical nephrectomy, $R C$ radical cystectomy, $c C R C C$ clear cell renal cell carcinoma, $p R C C$ papillary renal cell carcinoma, chRCC chromophobe renal cell carcinoma

may imply the increased susceptibility of urological malignancy in dialysis patients [11]. However, none of them were verified as specific risk factors for the simultaneous presence of tumors.

The golden standard for management of UTUC is radical nephroureterectomy, and the best choice of treatment for patients with synchronous ipsilateral RCC and UTUC seems to be radical nephroureterectomy. However, there is no question that partial nephrectomy has become a standard to manage small renal masses. Conservative management of UTUC has been discussed in low-risk cases and allows sparing the morbidity associated with radical surgery without compromising oncological outcomes and kidney function [12]. The 2015 European Association of Urology (EAU) guidelines suggest that conservative management may be considered when the tumor is unifocal, small $(<1 \mathrm{~cm})$, and low grade with no evidence of an infiltrative lesion on CT urography [13]. It therefore would appear justified to combine partial nephrectomy for RCC and kidneysparing management for UTUC in an appropriately selected patient. Benjamin et al. have reported a patient who received renal sparing management of his double malignancy, including open partial nephrectomy of his T1a RCC and endoscopic laser ablation of his low-grade Ta ipsilateral renal pelvis urothelial carcinoma. The patient is healthy with normal serum creatinine and followed with regular surveillance ureteroscopy after 4 years [14]. This option should therefore be discussed provided that both lesions would be suitable for renalpreserving surgery. For the patient with a small renal mass $(<7 \mathrm{~cm})$ and a unifocal, small $(<1 \mathrm{~cm})$, and lowgrade ipsilateral UTUC, partial nephrectomy of RCC and kidney-sparing management of UTUC may be combined together.

Different treatments were used to manage synchronous RCC and bladder cancer in our study. The treatment of bladder tumor was decided by tumor stage. Radical cystectomy was performed for muscle-invasive bladder tumor. For RCC, most cases were treated by partial nephrectomy. Radical nephrectomy did not improve overall survival. It is suitable to take renal-sparing surgery into consideration if RCC meets the indications for partial nephrectomy. Although none of cases with PN or with RN emerged with ureter carcinoma recurrence during follow-up, synchronous distal ureter carcinoma was detected in the pathological specimen of one patient treated with RNU. Urothelial carcinoma presents a feature of multifocality. Preoperative imaging examination should be meticulous to obtain as much information as possible and to ensure the identification of suspicious masses for patients with concurrent RCC and UC.

One patient was diagnosed as RCC and concomitant contralateral ureter carcinoma in our study. In order to avoid the risk of renal failure after PN and contralateral radical surgery, we combined bilateral renal-preserving surgery and segmental ureteral resection with ureteroureteral anastomosis was performed for contralateral ureter carcinoma. Bladder tumor occurred after 1 year and TURBT was performed. He was still followed with regular surveillance. So far, only around 10 cases of RCC with synchronous contralateral UTUC have been described in the literature [15]. Opinions vary on proper treatment of synchronous bilateral renal tumors of different histogenesis, and no general guideline has been set. For the renal cell carcinoma, radical nephrectomy, partial nephrectomy, tumor enucleation, and angioinfarction were reported. For the renal pelvis tumor, nephroureterectomy, partial nephrectomy, radical nephrectomy. and tumor excision were reported. We believe that management plans must be individualized. All the factors including biological features of each tumor, bilateral renal function, and life quality of the patient should be considered together. 
A review of 47 cases of renal tumors reported that the overall prognosis of synchronous renal tumors were not worse than isolated ones [7]. Recently, Dutta et al. have shown that the prognosis for a patient with dual malignancies is likely most influenced by the more aggressive one of the two tumors [5]. In our study, four patients died of RCC. These patients had higher stage and grade of RCC than their urothelial carcinoma, and one of them had multiple bone metastases from RCC before surgery. One patient died of ureter carcinoma 3 months after surgery with postoperative pathology of high-grade urothelial carcinoma with squamous and sarcomatous differentiation. Our result is in accordance with the hypothesis that the prognosis is possibly associated with the more aggressive one.

\section{Conclusions}

The incidence of synchronous tumors may increase simultaneously with the increased incidence of genitourinary tumors. It is necessary to pay attention to the possible occurrence of synchronous urothelial cancer in clinical practice. Treatments should be individualized. The biological features of each tumor and bilateral renal function of the patient must be considered comprehensively. The prognosis is possibly associated with the more aggressive one. Further follow-up will combine aspects of recommended follow-up for both RCC and urothelial carcinoma, as per published guidelines.

\section{Abbreviations}

CIS: Carcinoma in situ; CT: Computed tomography; PN: Partial nephrectomy; RC: Radical cystectomy; RCC: Renal cell carcinoma; RN: Radical nephrectomy; RNU: Radical nephroureterectomy; TURBT: Transurethral resection of bladder tumor; UTUC: Upper tract urothelial carcinoma

\section{Acknowledgements}

Not applicable.

\section{Funding}

This work was supported by National Natural Science Foundation of China (No. 81502193), the Science and Technology Department of Jiangsu Province (No. BK20151167), and the Distinguished Talents of Six Domains in Jiangsu Province (No. WSW-064)

Availability of data and materials

Please contact author for data requests.

\section{Authors' contributions}

NQ and YC collected surgical data, reviewed related literatures, and drafted the article. KG processed the data and the images. $\mathrm{HL}$ is the corresponding author who designed the study and revised the article. All authors read and approved the final manuscript.

\section{Ethics approval and consent to participate}

Informed consent was obtained from the patient for the publication of this report and any accompanying images. The study was approved by the institutional review board from Peking University First Hospital and The Affiliated Hospital of Xuzhou Medical University.

\section{Consent for publication}

Written informed consent was obtained from the patient for the publication of this report.

\section{Competing interests}

The authors declare that they have no competing interests.

\section{Publisher's Note}

Springer Nature remains neutral with regard to jurisdictional claims in published maps and institutional affiliations.

\section{Author details}

'Department of Urology, The Affiliated Hospital of Xuzhou Medical University, Xuzhou, China. ${ }^{2}$ Department of Urology, Peking University First Hospital, Beijing, China. ${ }^{3}$ Institute of Urology, Peking University, Beijing, China.

${ }^{4}$ National Urological Cancer Center, Beijing, China.

Received: 27 November 2017 Accepted: 19 January 2018

Published online: 25 January 2018

\section{References}

1. Graves RC, Templeton ER. Combined tumors of the kidney. J Urol. 1921;5: $517-37$.

2. Siegel RL, Miller KD, Jemal A. Cancer statistics. CA Cancer J Clin. 2015;65:5-29.

3. Mucciardi G, Gali A, D'Amico C, et al. Transitional cell carcinoma of the renal pelvis with synchronous ipsilateral papillary renal cell carcinoma: case report and review. Case Rep Urol. 2015;3:93-5.

4. Atilgan D, Uluocak N, Parlaktas BS. Renal cell carcinoma of the kidney with synchronous ipsilateral transitional cell carcinoma of the renal pelvis. Case Rep Urol. 2013:2013:194127.

5. Dutta G, Silver D, Oliff A, et al. Synchronous renal malignancy presenting as recurrent urinary tract infections. Case Rep Urol. 2011;2011:832673.

6. Leveridge $\mathrm{M}$, Isotalo PA, Boag AH, et al. Synchronous ipsilateral renal cell carcinoma and urothelial carcinoma of the renal pelvis. Can Urol Assoc J. 2009;3:64-6.

7. Fernández Arjona M, Santos Arrontes D, De Castro Barbosa F, et al. Synchronous renal clear-cell carcinoma and ipsilateral transitional-cell carcinoma: case report and bibliographic review. Arch Esp Urol. 2005:58:460-3.

8. Lu Q, Zhuang J, Guo H. Renal cell carcinoma with synchronous ipsilateral urothelial carcinoma of the renal pelvis. Oncol Lett. 2017:13(6):4521-5.

9. Oka H, Kobayashi S, Kobayashi $\mathrm{T}$, et al. Multiple primary cancers limited to the urological field. Hinyokika Kiyo. 2001;47(6):405-9.

10. Demir A, Onol FF, Bozkurt S, et al. Synchronous ipsilateral conventional renal cell and transitional cell carcinoma. Int Urol Nephrol. 2004;36:499-502.

11. Lee CT, Lin JW, Hsu K, et al. Concurrent renal cell carcinoma and transitional cell carcinoma in a chronic hemodialysis patient. Ren Fail. 2003;25:863-9.

12. Yakoubi R, Colin P, Seisen T, et al. Radical nephroureterectomy versus endoscopic procedures for the treatment of localized upper tract urothelial carcinoma: a meta-analysis and a systematic review of current evidence from comparative studies. Eur J Surg Oncol. 2014:40:1629-34.

13. Rouprêt $M$, Babjuk $M$, Compérat $E$, et al. European association of urology guidelines on upper urinary tract urothelial cell carcinoma: 2015 Update. Eur Urol. 2015;68:868-79.

14. Bay BN, Black P. Double jeopardy? Renal-sparing management of simultaneous ipsilateral renal cell carcinoma and urothelial carcinoma. Can Urol Assoc J. 2013;7:e508-10.

15. Hong SK, Jeong SJ, Lee SE. A case of renal transitional cell carcinoma associated with synchronous contralateral renal cell carcinoma. J Korean Med Sci. 2001;16:108-10 